\title{
Golfo Dulce, Costa Rica, un área importante de alimentación para la tortuga carey del Pacífico Oriental (Eretmochelys imbricata)
}

\author{
Didiher Chacón-Chaverri ${ }^{1}$, David A. Martínez-Cascante ${ }^{2}$, David Rojas ${ }^{1} \&$ Luis G. Fonseca $^{1}$ \\ 1. WIDECAST Costa Rica, Tibás, 496-1100, San José, Costa Rica; dchacon@widecast.org, rojasmoralesd78@gmail.com, \\ luisfonsecalopez@gmail.com \\ 2. San Josecito de San Rafael, Heredia, Costa Rica; dawidh15@gmail.com
}

Recibido 11-VIII-2014. Corregido 20-XI-2014. Aceptado 22-XII-2014.

\begin{abstract}
Golfo Dulce, Costa Rica, an important foraging ground for the Pacific hawksbill turtle (Eretmochelys imbricata). Limited quantitative information is available for hawksbill turtles (Eretmochelys imbricata) at foraging grounds in the Eastern Tropical Pacific (ETP), where the species composes one of the most endangered marine turtle populations on the planet. Between August 2010 and March 2013 we captured individual hawksbill turtles using entanglement nets along the edges of mangroves and seagrasses of the Golfo Dulce, in southwest Pacific Costa Rica. A total of 62 hawksbills were captured, including 14 recaptures, of which $46(74.19 \%)$ were juveniles $(\mathrm{CCL}<66 \mathrm{~cm})$ and $16(25.81 \%)$ were adults. The catch per unit effort (1 unit: $100 \mathrm{~m}$ of net for $7 \mathrm{~h}$ ) during the study ranged between 0.03 and 0.07 . The Golfo Dulce is highly turbid during the rainy season (May-November), particularly at our study area, as high sediment loads due to intensive runoff lead to poor water clarity. The probability of detection of hawksbills was considerably higher in the dry season (December-April) compared to the rainy season, suggesting these turtles may prefer waters with higher clarity. None of the individuals captured had evidence of internal or external tags, making it possible to conclude that they had not been previously marked at other feeding or breeding sites. A total of $28(45.16 \%)$ individuals were found to host the ectoparasitic barnacle Stephanolepas muricata, which in high concentrations can be harmful by limiting the mobility of organs and limbs. Although consistent in-water quantification of hawksbills in the ETP remains scant, this study represents the longest and most robust marine monitoring dataset for hawksbills in the region to date. Our findings highlight the relevance of the Golfo Dulce as an important foraging ground for hawksbill turtles in the ETP and emphasize the need to monitor and protect this habitat to aid efforts to recover this critically endangered marine turtle population. Rev. Biol. Trop. 63 (Suppl. 1): 351-362. Epub 2015 April 01.
\end{abstract}

Key words: Golfo Dulce, hawksbill turtle, Eretmochelys imbricata, Costa Rica, entanglement nets, sea grass.

La tortuga carey es una especie catalogada en peligro crítico por la Unión Internacional para la Conservación de la Naturaleza (UICN) (UICN, 2012) y se encuentra incluida en el Apéndice I de la Convención sobre el Comercio Internacional de Especies Amenazadas de Fauna y Flora Silvestres (CITES) (CITES, 2012). Hasta hace pocos años, el estado de conservación de esta especie en el Pacífico Oriental Tropical era desconocido, con eventos de anidación aislados en algunos sitios de México y Centroamérica (Meylan \& Donnelly, 1999). Sin embargo, desde mediados del 2008 comenzaron a descubrirse sitios importantes de anidación, particularmente en El Salvador y Nicaragua donde alberga aproximadamente $80 \%$ de la población anidadora del Pacífico Oriental (Gaos et al., 2010; Liles et al., 2011; Altamirano, 2010) y donde se han establecido programas de conservación de nidadas. También se han descubierto sitios de anidación relativamente pequeña en donde se están protegiendo nidadas de carey, en países como México y Ecuador (Gaos et al., 2010). A pesar de que estas acciones de protección pueden colaborar con la recuperación de la especie en 
la región, se estima que menos de 300 hembras anidadoras existen en todo el Pacífico Oriental (Gaos et al., 2012a), resaltando la vulnerabilidad de la población, la cual actualmente es considerada una de las siete poblaciones de tortugas marinas más amenazadas del mundo (Wallace et al., 2011).

En el caso particular de Costa Rica, la anidación de tortuga carey aparece esporádicamente a lo largo de la costa Pacífica. Se han reportado hembras anidadoras en el Pacífico Norte en playas como Isla San José, Lagartillo, Avellanas, Callejones, Punta Banco, Caletas y Caña Blanca, sin embargo, en ninguno de los sitios se superan las cinco nidadas por temporada (Gaos, Arauz \& Yañez, 2006; Ward, Elkins \& Ward, 2010; Fonseca, Quirós, Villachica, Mora, Heidemeyer \& Valverde, 2013 com. pers.). En el Pacífico Sur de Costa Rica, la anidación se restringe a las playas Sombrero, Tamales y Platanares, en donde anualmente se contabilizan menos de 25 nidadas por temporada (Chacón-Chaverri, observ. pers).

Al contrario de los esfuerzos realizados en playas de anidación en donde se ha monitoreado a las hembras y nidadas por décadas (e.g. Playa Nancite y Playa Grande) (SaltidriánTomillo, Saba, Piedra, Paladino \& Spotila, 2008; Fonseca, Murillo, Guadamúz, Spínola, \& Valverde, 2009), la información en Costa Rica sobre sitios de alimentación o áreas de agregación de tortugas carey en el agua es escasa en Costa Rica. En Guanacaste existe información para un área de Punta Coyote y en Punta Pargos. Para la primera zona se determinó que las tortugas en su mayoría presentaron fidelidad al sitio (Carrión, 2010), y que tenían preferencia de alimentación de esponjas (Geodia sp.) y de ascidias (Rhopalaea birkelandi) (CarriónCortés, Canales-Cerro, Arauz \& RiosmenaRodríguez, 2013). Frente a las costas de Punta Pargos existe evidencia sobre la presencia de individuos juveniles o sub-adultos los cuales han salido muertos a la playa, posiblemente como consecuencia de la interacción con artes de pesca (Ward et al., 2010). En el caso del Golfo Dulce, no existe ninguna información técnica ni anecdótica sobre la presencia de la tortuga carey, tanto en zonas de alimentación como en sitios de anidamiento.

Dado esta falta de información técnica y científica sobre la especie, este estudio se dio a la tarea de colectar información sobre las tortugas carey que se agrupan en el sector oeste del Golfo Dulce, el cual es un área conformada por manglares, arrecifes de coral, pastos marinos $\mathrm{y}$ fondos lodosos y rocosos, los cuales ofrecen condiciones apropiadas para la alimentación de la tortuga carey.

El objetivo de este estudio es demostrar la presencia de individuos de tortuga carey en el sector oeste del Golfo Dulce, para lo cual evaluamos la probabilidad de presencia temporal y estacional, así como la descripción de la estructura de la población. Esta información es fundamental para entender el uso de los hábitats y la abundancia de la tortuga carey en Costa Rica, así como el papel de la costa Pacífica del país en la disponibilidad de sitios de forrajeo para la población a escala regional.

\section{MATERIALES Y MÉTODOS}

Área de estudio: El estudio se realizó en las aguas costeras de Playa Blanca, en el sector oeste del Golfo Dulce, el cual se ubica en el cantón de Osa de la Provincia de Puntarenas, Costa Rica $\left(8^{\circ} 38^{\prime} 51^{\prime \prime} \mathrm{N}-83^{\circ} 25^{\prime} 54^{\prime \prime}\right.$ W) (Fig. 1). El Golfo Dulce se considera como un fiordo tropical y sus condiciones oceanográficas y geomorfológicas únicas permiten el desarrollo de ecosistemas particulares (Cortés, 1992). El sector oeste del golfo esta bordeado por manglares y bosques tropicales que están interconectados por las desembocaduras de los ríos o quebradas, los cuales son influenciados fuertemente por las mareas. En este sitio están presentes dos especies de pastos marinos: Halophyla sp. y Halodule sp., así como tres especies de mangle: Rhizophora mangle, $R$. racemosa, Avicennia germinans. Además, en el Golfo Dulce se presentan parches de corales, principalmente dominados por Porites lobata. Actualmente la cobertura de coral vivo es menor al $10 \%$, debido principalmente a la sedimentación, deforestación de las cuencas y 


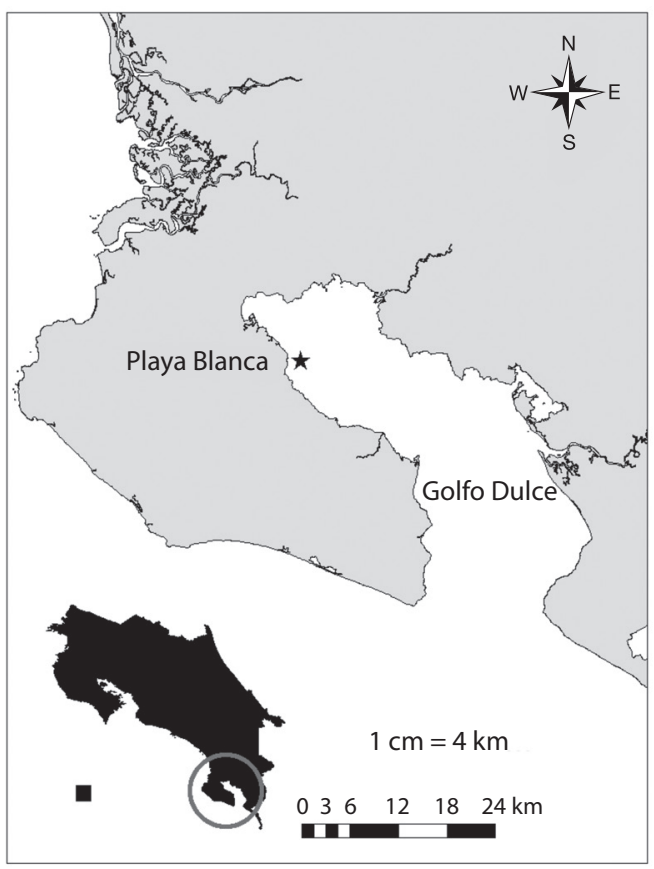

Fig. 1. Mapa de ubicación de Playa Blanca, Golfo Dulce, Costa Rica.

Fig. 1. Location map of Playa Blanca, Golfo Dulce, Costa Rica.

fenómenos naturales como huracanes (Fonseca, Dean \& Cortés, 2006).

En esta área la precipitación muestra un patrón bimodal, con un período de altas lluvias desde mayo hasta noviembre, y un período seco entre enero y abril. El promedio de precipitación mensual desde el año 2000 ha oscilado entre los $3500 \mathrm{~mm}$ y $5500 \mathrm{~mm}$, con una temperatura anual promedio de $26^{\circ} \mathrm{C}$ (Instituto Meteorológico Nacional, 2014).

Desde el punto de vista del manejo y uso de los recursos pesqueros, el Golfo Dulce es un sitio declarado por el Instituto Costarricense de Pesca y Acuacultura (INCOPESCA) como un Área Marina de Pesca Responsable (AMPR). Este Decreto Ejecutivo fue firmado en agosto de 2010 y el área abarca unos $750 \mathrm{~km}^{2}$. Dentro de esta AMPR se prohíbe la pesca de arrastre, palangre y trasmallo. Además de regular el tipo de anzuelo y su tamaño.
Captura de las tortugas marinas y CPUE: El periodo de estudio abarcó desde agosto de 2010 hasta marzo de 2013, en donde se implementaron redes de enmalle $(100 \times 8 \mathrm{~m}$, luz de malla $=50 \mathrm{~cm}$ ) para capturar las tortugas. Las redes fueron colocadas únicamente durante el día, durante periodos de $7 \mathrm{~h}$, tratando iniciar el monitoreo a las 8:00h de cada día de muestreo. La unidad de esfuerzo se definió como el despliegue de $100 \mathrm{~m}$ de la red de enmalle durante $7 \mathrm{~h}$, la CPUE se calculó dividiendo el número total de tortugas marinas capturadas en cada muestreo dividido por el número de unidades de esfuerzo. La distancia entre la red y la costa varió entre los 100 y 500m de longitud, y entre $\operatorname{los} 3$ y $10 \mathrm{~m}$ de profundidad, dependiendo de los movimientos de las mareas y la precipitación. Luego de colocar la red, el bote se movilizó a una distancia no mayor a los $200 \mathrm{~m}$, para tener visibilidad de las boyas flotantes de la red. Las boyas permiten detectar cuando una tortuga es capturada, ya que las boyas tienen a bajar y no es posible observarlas. Posterior a la captura de una tortuga, esta liberada de la red, subida a una embarcación y llevada a la arena. Después de realizar las mediciones (ver abajo), la tortuga fue marcada con marcas metálicas Inconel (Tipo 681, National Band \& Tag Company, Newport, KY), específicamente sobre la segunda escama proximal de cada aleta delantera. Asimismo, desde enero de 2012 se colocó un PIT (Passive Integrated Transponder) en la aleta delantera derecha. Previo a la aplicación de las marcas metálicas, éstas fueron desinfectadas con Vanodine (Pfizer Inc.) para evitar infecciones en la piel de la tortuga e incrementar la coagulación de la sangre y la velocidad de cicatrización. El personal de campo siempre utilizó guantes de látex durante la manipulación de la tortuga, por previsiones de bioseguridad.

Biometría del caparazón: El largo curvo de caparazón (LCC) fue medido utilizando una cinta métrica flexible $( \pm 0.1 \mathrm{~cm})$. Cada medición se realizó por triplicado para verificar cada una de las medidas. Se realizó una clasificación de cada tortuga de acuerdo al tamaño del LCC 
para determinar la estructura de la población. Se consideró que a las tortugas que presentaban un LCC $\geq 66 \mathrm{~cm}$ como adultos, mientras que los individuos que presentan una medición menor se clasificaron como juveniles. Esto debido a que $66 \mathrm{~cm}$ de LCC es la menor medida reportada para una hembra anidando en El Salvador y Nicaragua (Altamirano, 2010; Altamirano \& Torres, 2011; Liles et al., 2011; Gaos et al., 2012a; Torres \& Altamirano, 2012).

Evaluación corporal: A cada tortuga capturada se le realizó una evaluación ocular para detectar lesiones, amputaciones, deformidades o la presencia de fibropapilomas o ectoparásitos. En los casos en donde se detectó alguna de estas características, está fue descrita en la hoja de campo. Las tortugas que presentaron más de 10 parásitos externos en su piel del cirripedio Stephanolepas muricata, fueron llevadas al centro de rescate de WIDECAST en donde fueron colocadas por $24 \mathrm{~h}$ en un estanque de agua dulce, para hidratar la piel de la tortuga y por desalinización matar al parásito. Posteriormente, con la ayuda de pinzas quirúrgicas se removió el parásito, y se aplicó vanodine en el área afectada para incrementar el tiempo de cicatrización de la piel.

Probabilidad de detección: Los valores de la CPUE anuales no pudieron ser comparados debido a que los datos fueron fuertemente asimétricos, dada la gran cantidad de ceros (no captura), lo que impide el uso de un ANDEVA (Wenger \& Freeman, 2008). Por lo tanto, los datos de las capturas se agruparon como presencia y ausencia, con el fin de estimar la probabilidad de detección. Esta información fue analizada mediante una regresión logística ajustada por máxima verosimilitud (Bolker, 2008). Las variables independientes analizadas fueron: estación del año (seca o lluviosa), año del estudio (de 2010 a 2013), la cual fue estadarizada como a' $=\mathrm{a}-\min$ (a). Se compararon. Se compararon 4 modelos posibles: M1 es el modelo nulo, M2 analiza tendencias temporales, M3 analiza estacionalidad, M4 analiza tendencia temporal y estacionalidad simultáneamente. Los datos fueron analizados en el software R ( R Development Core Team, 2013), utilizando la rutina "glm", con distribución binomial y enlace "logit". Para la comparación de modelos se utilizó el Criterio de Información de Akaike (AIC) (Burnham \& Anderson, 2002).

\section{RESULTADOS}

Captura de las tortugas marinas y CPUE: Entre agosto de 2010 y marzo de 2013 se capturaron un total de 62 tortugas empleando una unidad de muestreo de 1078 (100m de red por $7 \mathrm{~h}$ ) (Cuadro 1). La CPUE se ha incrementado ligeramente a través de los años, llegando a duplicarse desde el 2010. La

CUADRO 1

Estadísticas de captura de la tortuga carey (Eretmochelys imbricata) en el Golfo Dulce, Costa Rica; incluyendo el número de tortugas marcadas, número de recapturas, tasa de recaptura y el esfuerzo de muestreo

TABLE 1

Catch statistics of the hawksbill turtle (Eretmochelys imbricata) in the Golfo Dulce, Costa Rica, including the number of turtles tagged, number of recaptures, recapture rate and sampling effort

\begin{tabular}{lccccccccc} 
Año & Tortugas & \multicolumn{2}{c}{ Tortugas recapturadas $(\mathrm{n}=14)$} & \multicolumn{2}{c}{ Tasa de } \\
marcadas & 2010 & 2011 & 2012 & 2013 & recaptura (\%) & Esfuerzo total de muestreo & CPUE \\
\hline 2010 & 6 & 2 & 2 & 1 & 0 & 83.33 & 189 & 0.03 \\
2011 & 5 & - & 0 & 1 & 1 & 40.00 & 105 & 581 & 0.05 \\
2012 & 37 & - & - & 2 & 5 & 18.92 & 203 & 0.06 \\
2013 & 14 & - & - & - & 0 & 0.00 & 1078 & 0.07 \\
Total & 62 & 2 & 2 & 4 & 6 & 22.58 & & 0.05 \\
\hline
\end{tabular}


tasa de recaptura ha disminuido a través de los años, hasta llegar a cero durante el 2013. El mayor número de tortugas se capturaron en el 2012 en donde igualmente se aplicó el mayor esfuerzo de muestreo. De las tortugas recapturadas, cinco correspondieron a hembras y tres a juveniles (Cuadro 2). El intervalo promedio de recaptura fue de 128.29 días (rango=8-625, $\mathrm{n}=14$ ), siendo una hembra la que más tiempo ocupó el sitio de muestreo con 799 días. Un individuo se recapturó en tres ocasiones, cuatro en dos ocasiones y tres en una sola oportunidad. De las 14 tortugas recapturadas, se observaron tres que perdieron alguna de las marcas metálicas, lo que representa el 21.43\% de las capturas. Sin embargo, estas tortugas que perdieron alguna marca metálica conservaron la otra, por lo que no se recontaron individuos. Dos correspondieron a la aleta derecha y uno a la aleta izquierda. Ninguna de las tortugas capturadas poseía marcas previas, ni fue reportada anidando o varadas en alguna playa del Pacífico Oriental Tropical.

Biometría del caparazón: El LCC de las tortugas capturadas osciló entre 34.8 y $81.1 \mathrm{~cm}$ (LCC promedio $=56.5 \mathrm{~cm} \pm 5.3$ IC al 95\%) (Fig. 2). Según el LCC de las tortugas capturadas, $46(74.19 \%)$ correspondieron a individuos con tallas juveniles y $16(25.81 \%)$ a adultas (Fig. 2). Las clases dominantes fueron 50.0-54.9 y 60.0-64.9 con 12 tortugas cada una.
Evaluación corporal: Ninguna de las tortugas capturadas presentaron evidencia externa de fibropapilomas, traumas o lesiones en huesos, ojos y cloaca. Sin embargo, 28 de las 62 tortugas marcadas presentaron el cirripedio Stephanolepas muricata parasitando la piel de los individuos. En la mayoría de los casos el parásito se encontró en las aletas y la zona perianal.

Probabilidad de detección: Según el AIC, el modelo con el mejor desempeño fue el M3, el cual toma en cuenta la estacionalidad en relación a la probabilidad de detección de la tortuga carey en el Golfo Dulce. En caso de la ponderación de modelos, M3 cuenta con un $66 \%$ del peso entre los cuatro modelos evaluados. Sin embargo, el modelo M4 aporta un significativo $32 \%$ del peso, por lo cual merece atención (Cuadro 3).

El modelo M3 indica que la probabilidad de detectar una tortuga carey se incrementa durante la estación seca (Fig. 3), independientemente del año de estudio. El modelo M4, señala lo mismo, la estación seca incrementa la probabilidad de detectar una tortuga carey, sin embargo, también señala que esta probabilidad ha ido incrementando a través del periodo de estudio. No obstante, los intervalos de confianza del efecto del tiempo $\beta_{\text {año }}$ son amplios, abarcando incluso, una tendencia temporal negativa (Cuadro 3, Fig. 4).

\section{CUADRO 2}

Historia de recaptura de la tortuga carey (Eretmochelys imbricata), Golfo Dulce, Costa Rica

TABLE 2

Recapture history of the hawksbill turtle (Eretmochelys imbricata), Golfo Dulce, Costa Rica

\begin{tabular}{|c|c|c|c|c|c|c|c|c|}
\hline $\begin{array}{c}\text { Aleta } \\
\text { derecha }\end{array}$ & Estado & $\begin{array}{c}\text { Fecha de } \\
\text { captura }\end{array}$ & $\begin{array}{c}\text { Fecha de } \\
\text { recaptura \#1 }\end{array}$ & $\begin{array}{l}\text { PEPR } \\
\text { (Días) }\end{array}$ & $\begin{array}{c}\text { Fecha de } \\
\text { recaptura } \# 2\end{array}$ & $\begin{array}{l}\text { PEPR } \\
\text { (Días) }\end{array}$ & $\begin{array}{c}\text { Fecha de } \\
\text { recaptura \#3 }\end{array}$ & $\begin{array}{l}\text { PEPR } \\
\text { (Días) }\end{array}$ \\
\hline NG956 & Juvenil & 02-Oct-10 & 02-Nov-10 & 31 & 18-Ene-12 & 108 & - & - \\
\hline NG962 & Juvenil & 02-Oct-10 & 11-Dic-10 & 70 & - & - & - & - \\
\hline NG966 & Juvenil & 16-Oct-10 & 16-Set-11 & 335 & 19-Ene-12 & 452 & - & - \\
\hline PC976 & Adulto & 29-May-12 & 21-Jul-12 & 53 & - & - & - & - \\
\hline PE237 & Adulto & 20-Jan-11 & $06-O c t-12$ & 625 & 30-Mar-13 & 799 & & \\
\hline GL783 & Adulto & 09-Sep-12 & 20-Oct-12 & 41 & 03 -Ene-13 & 115 & 19-Ene-13 & 130 \\
\hline GL704 & Adulto & 21-Sep-12 & 28-Ene-13 & 129 & 11-Mar-13 & 170 & - & - \\
\hline NG886 & Adulto & 14-Feb-12 & 22-Feb-13 & 8 & - & - & - & - \\
\hline
\end{tabular}




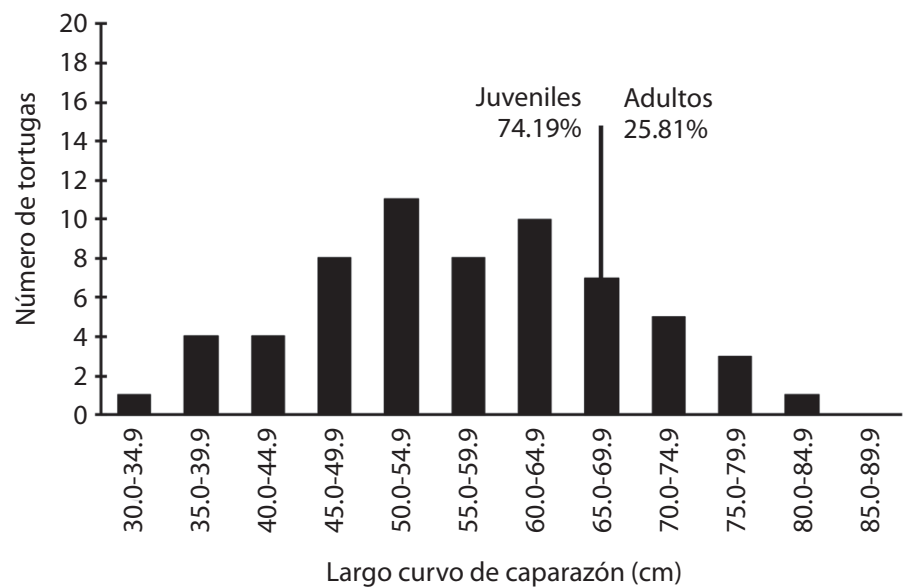

Fig. 2. Histograma del largo curvo de caparazón (LCC) de las tortugas carey (Eretmochelys imbricata) capturadas en el Golfo Dulce, Costa Rica entre agosto de 2010 y marzo de 2013. Solamente incluye las tortugas capturas en la primera ocasión, y se muestra la relación entre el porcentaje de juveniles y adultos capturados.

Fig. 2. Histogram of curved carapace length (CCL) of hawsbill turtles (Eretmochelys imbricata) captured in the Golfo Dulce, Costa Rica between August 2010 and March 2013. Only includes turtle catches in the first time, and shows the relationship between the percentage of juveniles and adults captured.

CUADRO 3

Comparación de modelos para estimación de la probabilidad de captura de la tortuga carey (Eretmochelys imbricata) en el Golfo Dulce, Costa Rica

TABLE 3

Comparison of models to estimate the probability of capture of the hawksbill turtle (Eretmochelys imbricata) in the Golfo Dulce, Costa Rica

\begin{tabular}{|c|c|c|c|c|c|c|c|}
\hline & Modelo & $\beta_{0}$ & $\beta_{\text {año }}$ & $\beta_{\text {seca }}$ & AIC & $\mathrm{dAIC}$ & Peso \\
\hline M1: & $p()$. & $-0.524(-0.85,-0.2)$ & & & 207.89 & 8.81 & 0.00 \\
\hline M2: & $p(a \tilde{n} o)$ & $-1.53(-2.68,-0.49)$ & $0.36(0.01,0.73)$ & & 205.85 & 6.73 & 0.02 \\
\hline M3 & $p(e s t)$ & $-1.08(-1.60,-0.60)$ & & $1.11(0.44,1.79)$ & 199.07 & 0.00 & 0.66 \\
\hline M4 & $p(e s t+a \tilde{n} o)$ & $-1.42(-2.54,-0.38)$ & $0.14(-0.24,0.54)$ & $1.00(0.26,1-75)$ & 200.57 & 1.49 & 0.32 \\
\hline
\end{tabular}

$\beta_{0}$ Intercepto; $\beta_{\text {año }}$ tendencia temporal; $\beta_{\text {seca }}$ estacionalidad. AIC: Criterio de información de Akaike, para comparar modelos. dAIC :diferencia del modelo actual con el mejor modelo. Peso: evidencia relativa del modelo actual contra los otros modelos analizados.

\section{DISCUSIÓN}

El uso de redes de enmalle para la captura de tortugas carey en el Pacífico Oriental ha sido una técnica poco utilizada. Únicamente se ha reportado su uso en Punta Coyote, Costa Rica, en donde se capturaron un total 17 tortugas, las cuales seis fueron recapturadas dos veces y una en tres ocasiones (Carrión-Cortés et al., 2013). Esta cifra es inferior si la comparamos con las 62 tortugas capturadas en el Golfo Dulce, no obstante, el esfuerzo invertido es mucho mayor en el Golfo Dulce (1.078h vrs 300h).

El Golfo Dulce, es un sitio reconocido por ser uno de los cuatro fiordos tropicales a nivel mundial, y se caracteriza por presentar condiciones topográficas que limitan la circulación del agua en su cuenca media y favorece la formación de un medio anóxico en las 


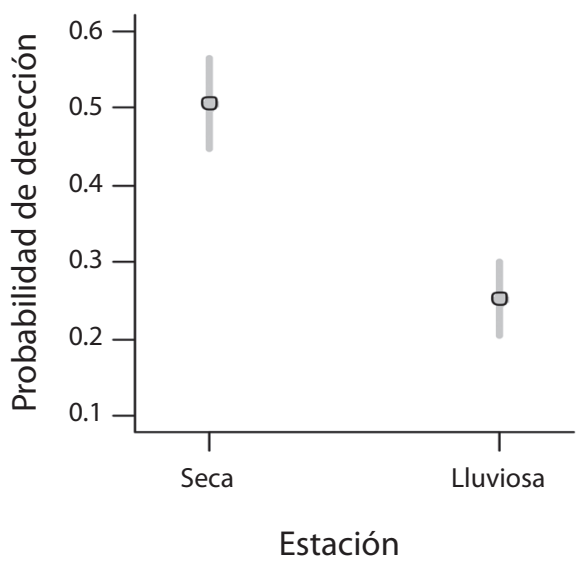

Fig. 3. Probabilidad de detección estacional para la tortuga carey (Eretmochelys imbricata) en el Golfo Dulce, según el modelo M3. Las barras grises representan el error estándar de los estimados.

Fig. 3. Seasonal detection probability for the hawksbill turtle (Eretmochelys imbricata) in the Golfo Dulce according to the model M3. The gray bars represent the standard error of the estimates.

profundidades (Quesada-Alpízar \& MoralesRamírez, 2004). El Golfo Dulce también se caracteriza por poseer áreas con parches coralinos en los bordes de la costa y su interior, los cuales se encuentran bastante deteriorados, como consecuencia de la sedimentación que es arrastrada desde el continente, fuertes periodos del Fenómeno del Niño, la deforestación y la minería (Cortés, 1992; Cortés, Jiménez, Fonseca \& Alvarado, 2010). Este tipo de sustrato normalmente es utilizado por individuos juveniles de tortuga carey en el Caribe y Australia, en donde se alimentan principalmente de esponjas calcáreas (Limpus, 1992; Meylan, 1988).

Normalmente, las tortugas en esta etapa de su ciclo de vida son residentes con un alto grado de fidelidad a su área de alimentación (van Dam \& Diez, 1997; Gaos et al., 2012a). Nuestros datos confirman este supuesto, como se observó en un juvenil capturada 452 días después de haber sido marcada. Asimismo, el Golfo Dulce presenta bosques de mangle y pastos marinos que son reconocidos como los hábitats predilectos de la tortuga carey en el Pacífico Oriental, especialmente en El Salvador y Nicaragua (Gaos et al., 2012a). Desde el 2010, el Golfo Dulce ha sido declarado como un AMPR por parte del INCOPESCA, por lo que en su interior no se permite el uso de artes de pesca como el palangre, trasmallo y arrastre. Esta medida propicia la protección de las tortugas carey, ya que estas artes han sido descritas como una de las principales amenazas para las tortugas marinas en el Pacífico Oriental
Seca

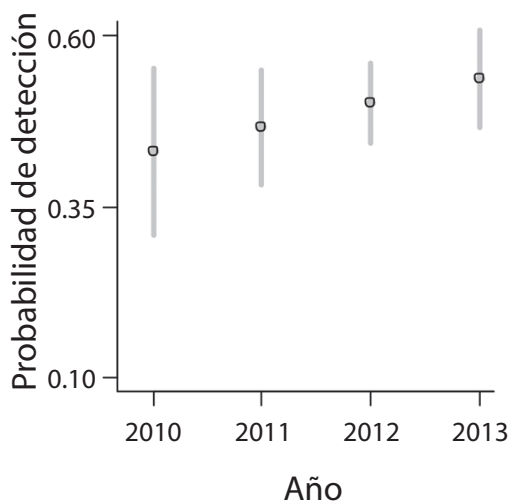

Lluviosa

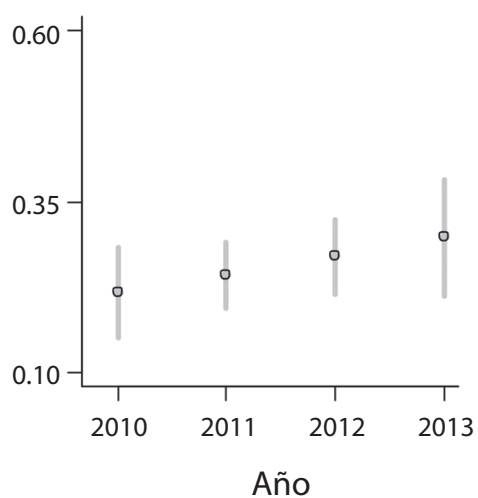

Fig. 4. Estimación de la probabilidad de detección temporal y estacional de la tortuga carey (Eretmochelys imbricata) según el modelo M4. Las barras grises representan el error estándar de los estimados (la estimación de la época lluviosa del 2013 corresponde a una predicción del modelo).

Fig. 4. Temporarily and seasonal estimation of detection probability by hawksbill turtle (Eretmochelys imbricata) according to model M4. The gray bars represent the standard error of the estimate (the estimate of the rainy season of 2013 corresponds to a prediction of the model). 
(Carranza, Domingo \& Estrades, 2006; Koch, Nichols, Peckham \& De la Toba, 2008).

Los datos recopilados en este estudio representan la segunda información sobre zonas de agregación de tortugas carey en el Pacífico de Costa Rica. En general, la información sobre esta especie es bastante limitada, como consecuencia de una reducción sostenida de las poblaciones en el Pacifico Oriental (Gaos et al., 2010; Liles et al., 2011) y los retos logísticos de estudiarlas en ambientes marinos. Previamente se describió que las tortugas carey de Punta Coyote se alimentan primariamente de esponjas (Geodia sp.), ascidias (Rhopalaea birkelandi), macroalgas, crustáceos y cnidarios (Carrión-Cortés et al., 2013), especies que pueden eventualmente servir de alimento a las tortugas carey en el Golfo Dulce, sin embargo, se requiere de estudios sobre la dieta de las tortugas en este sitio.

Por otro lado, en Punta Coyote se encontró que el $92 \%$ de las tortugas capturadas correspondieron a individuos juveniles (CarriónCortés et al., 2013), lo cual es superior a lo observado en este estudio en donde este valor fue del $74.19 \%$, lo que sugiere que en el Golfo Dulce los adultos confluyen en un porcentaje mayor. Esto último es difícil de documentar en esta especie, ya que la gran mayoría de estudios en zonas de alimentación raramente se capturan adultos, e incluso las tortugas no superan los 60cm (Boulon, 1994; Seminoff, Nichols, Resendiz \& Brooks, 2003; Blumenthal et al., 2009; Carrión-Cortés et al., 2013).

En el Pacífico Oriental, los primeros indicios sobre las zonas de alimentación de las hembras anidadoras de Nicaragua y El Salvador fueron derivados de transmisores satelitales colocados en las playas de anidación, en donde observó que las tortugas prefieren zonas neríticas, con influencia de manglares y pastos marinos en los alrededores del Golfo de Fonseca (Gaos et al., 2012a). En general nuestras observaciones coinciden con lo sugerido por Gaos et al. (2012b), en donde ellos indican que debido a la escasez de arrecifes de coral en el Pacífico Oriental, la tortuga carey ha diversificado su dieta, utilizando los recursos disponibles en los manglares. Esto previamente había sido aducido por Bjorndal y Bolten (2010), cuando consideran que la preferencia de la tortuga carey por utilizar pastos marinos en el Caribe, es una respuesta a la reducción en la cantidad y calidad de los arrecifes coralinos. Es probable que en el caso del Golfo Dulce, la tortuga carey se esté aprovechando de la riqueza de macro y micro-invertebrados marinos asociados a los pastos marinos, en donde sin desplazarse grandes distancias y con poco esfuerzo de captura pueden acceder al alimento.

La CPUE en este estudio no fue un buen parámetro para evaluar la abundancia entre años, dado que los datos presentaron fuerte asimetría, debido al gran número de ocasiones en que no se capturaron individuos. Sin embargo, la CPUE general es un excelente parámetro para evaluar la abundancia entre distintas área de agrupamiento de tortugas carey (CarriónCortés et al., 2013). En Punta Coyote se estimó una CPUE de 0.03, lo cual es ligeramente inferior a lo registrado en Golfo Dulce.

En cuanto a la evidencia sobre una tendencia temporal en la probabilidad de detección, notamos un comportamiento bimodal, ya que durante los dos primeros años de muestreo se capturaron pocas tortugas. Esto cambio durante los dos últimos años en donde incluso en el 2011 se capturaron 37 individuos. Sin embargo, no es clara la razón por la cual se dio este incremento, ya que se ha aplicado la misma metodología de muestreo a través del tiempo. Además, no se ha variado el área ni las horas de muestreo. Por tanto, se requiere la continuación del presente estudio por un periodo más largo, para tener una ventana de tiempo más apropiada para realizar conclusiones sobre el estado de la población en el Golfo Dulce.

Estacionalmente los datos sugieren que el sector oeste del Golfo Dulce es utilizado por las tortugas con una mayor frecuencia durante la época seca. Esto probablemente se debe a las tortugas prefieren ocupar hábitats con aguas cristalinas, como generalmente ocurre en los arrecifes coralinos. Es posible que durante la época lluviosa, el acarreo de sedimentos por escorrentía provoqué aguas turbias, lo que 
desemboque en que las tortugas se movilicen a otros sitios. Sin embargo, en El Salvador, Honduras y Nicaragua, los rangos de hogar de la tortugas carey adultas, comprenden áreas con aguas bastante turbias (Gaos et al., 2012a; Gaos et al., 2012b), lo que no apoya nuestra sugerencia sobre la posible preferencia estacional de las tortugas en el Golfo Dulce. En áreas más alejadas de los trópicos, en donde las aguas se enfrían fuertemente durante el invierno si se observa una diferencia estacional importante en la presencia de tortugas, debido a que durante el invierno se reduce la cantidad de alimento disponible (Felger, Cliffton \& Regal, 1976; Koch, Brooks \& Nichols, 2007).

En cuanto a la evaluación corporal, destaca la ausencia de fibropapilomas externos en la piel de las tortugas capturadas, sin embargo, es alarmante el porcentaje de individuos afectados por el cirripedio Stephanolepas muricata. Este epibionte pertenece a la familia Platylepadidae y normalmente se encuentra asociado a tortugas marinas, serpientes marinas y peces (Frick et al., 2011). Este cirripedio se caracteriza por encapsularse en el tejido del huésped, haciendo crecer lentamente las paredes de su concha hasta crear zonas de anclaje profundas en la dermis (Zardus \& Balazs, 2007). Quizás este ectoparásito no sea mortal para la tortuga carey, pero en cantidades considerables podría traer problemas de movilidad en extremidades lo que los convertiría en individuos más propensos a depredadores. En Costa Rica, este cirripedio fue descrito por primera vez en un individuos de tortuga carey pescado incidentalmente en una línea de palangre en el Pacífico Central (Corrales-Gómez \& Herrera-Ulloa, 2012). En el Pacífico Oriental, este epibionte también ha sido identificado en individuos de tortuga lora (Lepidochelys olivacea) en Playa Ceuta, México (Sosa-Cornejo et al., 2012) y en tortuga verde en Baja California (Frick et al., 2011) y Ecuador (Quiñones, GonzálezCarman, Zeballos, Purca \& Mianzan, 2010).

Implicaciones en el manejo: A pesar de que en Costa Rica el registro de tortugas carey en agua y sitios de anidación es escaso, la información colectada en este estudio representa el conjunto de datos más importante de un sitio de agrupamiento para el Pacífico Tropical. Esto hace que en el Golfo Dulce se deban de plantear nuevas políticas de gestión que propicien la conservación de los hábitats de las tortugas carey a través del tiempo. Máxime que esta zona está siendo amenazada por el desarrollo inmobiliario, los monocultivos y la pesca ilegal. A esto deben de sumarse los efectos del cambio climático que pueden generar efectos negativos sobre todo el ecosistema. Sin embargo, la declaración del golfo como un AMPR es una excelente oportunidad para gestionar de manera más efectiva el ordenamiento territorial marino.

Sin embargo, el reto es grande dada la falta de recursos técnicos y financieros que afrontan las entidades del Gobierno que velar por el cumplimento de la legislación vigente. Científicamente este estudio debe de complementarse con herramientas tecnológicas como la telemetría satelital y la genética para tratar de conocer los orígenes de estas tortugas. Esto permitirá que las medidas de protección y conservación se realicen de forma regional, demostrando que las aguas costarricenses podrían ser un área de agrupamiento para tortugas de Panamá y Costa Rica, como conciencia de la cercanía entre ambos países. Además, en Costa Rica se debe de explorar otros sitios de alimentación de tortuga carey, esto con el objeto de promover medidas de conservación que eviten la degradación de sus hábitats y la caza de tortugas para utilizar su caparazón en la fabricación de artesanías o espuelas para peleas de gallos. Todas estas acciones contribuirán directamente sobre la recuperación de la tortuga carey en el Pacífico Oriental Tropical.

\section{AGRADECIMIENTOS}

Agradecemos el apoyo financiero de la NOAA, Conservación Internacional, JFF, PTES, NFWF, EcoTeach, NEAQ, NAIB e International Student Volunteer. Asimismo, agradecemos a los cientos de voluntarios que participaron en este estudio, sin los cuales no 
hubiera sido posible la realización del mismo. Este estudio se realizó bajo los permisos de investigación AJDIP/127-2011, AJDIP/2272011, AJDIP/250-2011 y AJDIP/481-2012 otorgados por la Secretaría Técnica del INCOPESCA. Se agradece el Proyecto Consolidación de Areas Marinas Protegidas del Sistema Nacional de Áreas de Conservación, Programa de Naciones Unidas para el Desarrollo y el Global Environment Facility (GEF) por su colaboración en la publicación.

\section{RESUMEN}

La información cuantitativa disponible es escasa para la tortuga carey (Eretmochelys imbricata) en zonas de alimentación en el Pacifico Oriental, donde esta especie representa una de las poblaciones de tortugas marinas más amenazadas del mundo. Entre agosto de 2010 y marzo de 2013 capturamos individuos de tortuga carey utilizando redes de enmalle en los bordes de los manglares y los pastos marinos del Golfo Dulce, Costa Rica. Un total de 62 tortugas carey fueron capturadas, incluyendo 14 recapturas, de las cuales $46(74.19 \%)$ fueron juveniles (LCC $<66 \mathrm{~cm})$ y 16 (25.81) fueron adultas. La captura por unidad de esfuerzo (CPUE, 1 unidad: $100 \mathrm{~m}$ de red por $7 \mathrm{~h}$ ) durante el estudio oscilo entre 0.03 y 0.07 . El Golfo Dulces es altamente turbio durante la época lluviosa (mayo-noviembre), particularmente en nuestra área de estudio, debido a la intensa escorrentía que acarrea sedimentos que producen aguas poco cristalinas. La probabilidad de detección de tortugas carey fue considerablemente superior en la época seca (diciembre - abril) comparado con la época lluviosa, lo que sugiere que las tortugas prefieren hábitats con aguas cristalinas. Ninguna de las tortugas capturadas presentaba marcas externas o internas que hicieran concluir que habían sido marcadas en otros sitios de alimentación o reproducción. Un total de $28(45.16 \%)$ individuos fueron hospederos de un ectoparásito (Stephanolepas muricata), el cual puede ser dañino en altas concentraciones al limitar la movilidad de órganos y extremidades. A pesar de que el registro de tortugas carey en agua sigue siendo escaso en el Pacífico Tropical, hasta la fecha este estudio representa el conjunto de datos de monitoreo más largo y robusto para la especie en la región. Nuestros resultados demuestran la relevancia del Golfo Dulce como sitio de alimentación importante para la tortuga carey en el Pacífico Oriental y enfatiza en la necesidad de monitorear y proteger este hábitat, para ayudar a la recuperación de esta población de tortugas marinas que esta críticamente amenazada.

Palabras clave: Tortuga carey, Costa Rica, Golfo Dulce, redes de enmalle, pastos marinos.

\section{REFERENCIAS}

Altamirano, E. J. (2010). Informe Preliminar Proyecto de Conservación de Tortuga Carey (Eretmochelys imbricata) en la RN Estero Padre Ramos, Temporada 2010. Nicaragua: Flora y Fauna Internacional.

Altamirano, E. J. \& Torres, P. (2011). Informe del Proyecto de Conservación de tortuga carey (Eretmochelys imbricata) en la RN Estero Padre Ramos, Nicaragua, Temporada 2011. Nicaragua: Fauna y Flora Internacional e ICAPO.

Blumenthal, J. M., Austin, T. J., Bell, C. D. L., Bothwell, J, B., Broderick, A. C., Ebanks-Petrie, G., ...Godley, B. J. (2009). Ecology of hawksbill turtles, Eretmochelys imbricata, on a western Caribbean foraging ground. Chelonian Conservation and Biology, 8, 1-10.

Bolker, B. M. (2008). Ecological Models and Data in R. New Jersey: Princeton University Press.

Boulon, R. H. (1994). Growth Rates of Wild Juvenile Hawksbill Turtles, Eretmochelys imbricata, in St. Thomas, United States Virgin Islands. Copeia, 1994, 811-814.

Burnham, K. P., \& Anderson, D. R. (2002). Model selection and multimodel inference: a practical informationtheoretic approach. New York: Springer-Verlag.

Carranza, A., Domingo, A., \& Estrades, A. (2006). Pelagic longlines: A threat to sea turtles in the Equatorial Eastern Atlantic. Biological Conservation, 131, 52-57.

Carrión, J. A. (2010). Área de actividad local, dieta e intensidad de uso del hábitat de forrajeo de la tortuga carey (Eretmochelys imbricata) en el Pacífico Norte de Costa Rica (Tesis de Maestría). Universidad Autónoma de Baja California Sur, La Paz, México.

Carrión-Cortés, J., Canales-Cerro, C., Arauz, R., \& Riosmena-Rodríguez, R. (2013). Habitat Use and Diet of Juvenile Eastern Pacific Hawksbill Turtles (Eretmochelys imbricata) in the North Pacific Coast of Costa Rica. Chelonian Conservation and Biology, 12, 235-245.

CITES. (2012). Convención sobre el Comercio Internacional de Especies Amenazadas de Fauna y Flora Silvestres. Apéndice I, II y III. Ginebra, Suiza. Retrieved from www.cites.org

Corrales-Gómez, N., \& Herrera-Ulloa, A. (2012). First Record of the Turtle Barnacle Stephanolepas muricata from the Pacific Coast of Costa Rica. Marine Turtle Newsletter, 135, 9-10.

Cortés, J. (1992). Los arrecifes coralinos de Golfo Dulce, Costa Rica: aspectos ecológicos. Revista de Biología Tropical, 40, 19-26.

Cortés, J., Jiménez, C. E., Fonseca, A. C., \& Alvarado, J. J. (2010). Status and conservation of coral reefs in Costa Rica. Revista de Biología Tropical, 58, 33:50. 
Felger, R. S., Cliffton, K., \& Regal, P. (1976). Winter dormancy in sea turtles: independent discovery and explotation in the Gulf of California, México by two local cultures. Science, 191, 283-285.

Fonseca, A. C., Dean, H. K., \& Cortés, J. (2006). Non-colonial macro-borers as indicators of coral reef status in the south Pacific of Costa Rica. Revista de Biología Tropical, 54, 101-115.

Fonseca, L. G., Murillo, G. A., Guadamúz, L., Spínola, R. M., \& Valverde, R. A. (2009). Downward but Stable Trend in the Abundance of Arribada Olive Ridley Sea Turtles (Lepidochelys olivacea) at Nancite Beach, Costa Rica (1971- 2007). Chelonian Conservation and Biology, 8, 19-27.

Frick, M. G., Zardus, J. D., Ross, A., Senko, J., Montano-Valdez, D., Bucio-Pacheco, B., \& Sosa-Cornejo, I. (2011). Novel records and observations of the barnacle Stephanolepas muricata (Cirripedia: Balanomorpha: Coronuloidea); including a case for chemical mediation in turtle and whale barnacles. Journal of Natural History, 45, 629-640.

Gaos, A. R., Arauz, R., \& Yañez, I. L. (2006). Hawksbill Turtles on the Pacific Coast of Costa Rica. Marine Turtle Newsletter, 112, 14.

Gaos, A. R., Abreu-Grobois, F. A., Alfaro-Shigueto, J., Amorocho, D., Arauz, R., Baquero, A.,...Zárate, P. (2010). Signs of hope in the eastern Pacific: international collaboration reveals encouraging status for a severely depleted population of hawksbill turtles Eretmochelys imbricate. Oryx, 44, 595-601.

Gaos, A. R., Lewison, R. L., Wallace, B. P., Yañez, I L., Liles, M. J., Nichols, W. J., ...Seminoff, J. A. (2012a). Spatial ecology of critically endangered hawksbill turtles Eretmochelys imbricata: implications for management and conservation. Marine Ecology Progress Series, 450, 181-194.

Gaos, A. R., Lewison, R. L., Yañez, I. L., Wallace, B. P., Liles, M. J., Nichols, W. J., ... Seminoff, J. A (2012b). Shifting the life-history paradigm: discovery of novel habitat use by hawksbill turtles. Biology Letters, 8, 54-56.

Instituto Meteorológico Nacional. (2014). Boletín Junio 2014. San José, Costa Rica.

Liles, M. J., Jandres, M. V., López, W. A., Mariona, G. I., Hasbún, C. R., \& Seminoff, J. A. (2011). Hawksbill turtles Eretmochelys imbricata in El Salvador: nesting distribution and mortality at the largest remaining nesting aggregation in the eastern Pacific Ocean. Endangered Species Research, 14, 23-30.

Koch, V., Brooks, L. B., \& Nichols, W. J. (2007). Population ecology of the green/black turtle (Chelonia mydas) in Bahía Magdalena, Mexico. Marine Biology, $153,35-46$
Koch, V., Nichols, W. J., Peckham, H., \& De la Toba, V. (2008). Estimates of sea turtle mortality from poaching and bycatch in Bahía Magdalena, Baja California Sur, Mexico. Biological Conservation, $128,327-334$.

Limpus, C. J. (1992). The hawksbill turtle, Eretmochelys imbrlcata, in Queensland: population structure within a southern Great Barrier Reef feeding ground. Wildlife Research, 19, 489-506.

Meylan, A. B. (1988). Spongivory in hawksbill turtles: A diet of glass. Science, 239, 393-395.

Meylan, A. B. \& Donnelly, M. (1999). Status Justification for Listing the Hawksbill Turtle (Eretmochelys imbricata) as Critically Endangered on the 1996 IUCN Red List of Threatened Animals. Chelonian Conservation and Biology, 3, 200-224.

Quesada-Alpízar, M. A., \& Morales-Ramírez, A. (2004). Comportamiento de las masas de agua en el Golfo Dulce, Costa Rica durante El Niño (1997 - 1998). Revista de Biología Tropical, 52, 95-103.

Quiñones, J., González-Carman, V., Zeballos, J., Purca, S., \& Mianzan, H. (2010). Effects of El Niño-driven environmental variability on black turtle migration to Peruvian foraging grounds. Hydrobiologia, 645, 69-79.

R Development Core Team. (2013). R: A language and environment for statistical computing. R Foundation for Statistical Computing, Vienna, Austria. ISBN 3-900051-07-0.

Saltidrián-Tomillo, P., Saba, V. S., Piedra, R., Paladino, F. V., \& Spotila, J. R. (2008). Effects of Illegal Harvest of Eggs on the Population Decline of Leatherback Turtles in Las Baulas Marine National Park, Costa Rica. Conservation Biology, 22, 1216-1224.

Seminoff, J. A., Nichols, W. J., Resendiz, A., \& Brooks, L. (2003). Occurrence of Hawksbill Turtles, Eretmochelys imbricata (Reptilia: Cheloniidae), near the Baja California Peninsula, Mexico. Pacific Science, 57, 9-16.

Sosa-Cornejo, I., Montaño-Valdez, D. I., Bucio-Pacheco, M., Enciso-Saracho, F., Sanchez-Zazueta, J. G., \& Fierros-Pérez, E. (2012). Determination of Epibionts of the Marine Turtle Lepidochelys Olivacea (Eschscholtz, 1829) Nesting in Ceuta Beach, Sinaloa, Mexico. Journal of Agricultural Science and Technology, B2, 1190-1194.

Torres, P. \& Altamirano, E. (2012). Informe de proyecto de conservación de tortuga carey (Eretmochelys imbricata) en la Reserva Natural Estero Padre Ramos, Nicaragua. Temporada 2012. Nicarargua: Fauna y Flora Internacional e ICAPO. 
UICN. (2012). IUCN Red List of Threatened Species. IUCN Global Species Programme. Red List Unit: Cambridge, UK. Retrieved from www.iucnredlist.org

van Dam, R. P., \& Diez, C. E. (1997). Diving behavior of immature hawksbill turtles (Eretmochelys imbricata) in a Caribbean reef habitat. Coral Reefs, 16, 133-138.

Wallace, B. P., DiMatteo, A. D., Bolten, A. B., Chaloupka, M. Y., Hutchinson, B. J., Abreu-Grobois, A., ... Mast R. (2011). Global Conservation Priorities for Marine Turtles. PLoS ONE, 6(9), e24510.

Ward, M. W., Elkins, C., \& Ward, R. J. (2010). Punta Pargos Green Turtle Monitoring and Protection Program of Costa Rica. 2011 (Technical Report). Costa Rica: Sea Turtles Forever.

Wenger, S. J. \& Freeman, M. C. (2008). Estimating species occurrence, abundance, and detection probability using zero-inflated distributions. Ecology, 89, 2953-2959.

Zardus, J. D. \& Balazs, G. H. (2007). Two previously unreported barnacles commensal with the green sea turtle, Chelonia mydas (Linnaeus, 1758), in Hawaii and a comparison of their attachment modes. Crustaceana, 80, 1303-1315. 